\title{
The DiaS trial: dialectical behavior therapy versus collaborative assessment and management of suicidality on self-harm in patients with a recent suicide attempt and borderline personality disorder traits - study protocol for a randomized controlled trial
}

Kate Andreasson ${ }^{1,5^{*}}$, Jesper Krogh ${ }^{1}$, Bent Rosenbaum² ${ }^{2}$ Christian Gluud ${ }^{3}$, David A Jobes ${ }^{4}$ and Merete Nordentoft ${ }^{1}$

\begin{abstract}
Background: In Denmark 8,000 to 10,000 people will attempt suicide each year. The Centre of Excellence in Suicide Prevention in the Capital Region of Denmark is treating patients with suicidal behavior, and a recent survey has shown that $30 \%$ of the patients are suffering from borderline personality disorder. The majority of patients (70\% to 75\%) with borderline personality disorder have a history of deliberate self-harm and $10 \%$ have a lifetime risk to die by suicide. The DiaS trial is comparing dialectical behavior therapy with collaborative assessment and management of suicidality-informed supportive psychotherapy, for the risk of repetition of deliberate self-harm in patients with a recent suicide attempt and personality traits within the spectrum of borderline personality disorder. Both treatments have previously shown effects in this group of patients on suicide ideation and self-harm compared with treatment as usual.

Methods/Design: The trial is designed as a single-center, two-armed, parallel-group observer-blinded randomized clinical superiority trial. We will recruit 160 participants with a recent suicide attempt and at least two traits of the borderline personality disorder from the Centre of Excellence in Suicide Prevention, Capital Region of Denmark. Randomization will be performed though a centralized and computer-generated approach that conceals the randomization sequence. The interventions that are offered are a modified version of a dialectical behavior therapy program lasting 16 weeks versus collaborative assessment and management of suicidality-informed supportive psychotherapy, where the duration treatment will vary in accordance with established methods up to 16 weeks. The primary outcome measure is the ratio of deliberate self-harming acts including suicide attempts measured at week 28. Other exploratory outcomes are included such as severity of symptoms, suicide intention and ideation, depression, hopelessness, self-esteem, impulsivity, anger, and duration of respective treatments.
\end{abstract}

Trial registration: Clinical Trial.gov: NCT01512602.

Keywords: suicide prevention, deliberate self-harm, borderline personality disorder traits, BPD, dialectical behavior therapy, DBT, collaborative assessment and management of suicidality, CAMS

\footnotetext{
* Correspondence: kate.trein.andreasson@regionh.dk

${ }^{1}$ Mental Health Centre Copenhagen, Faculty of Health Science, University of

Copenhagen, Copenhagen, Denmark

${ }^{5}$ Research Unit, Mental Health Centre, Bispebjerg bakke 23, Building 14,

DK-2400 Copenhagen, NV, Denmark

Full list of author information is available at the end of the article
} 


\section{Background}

In Denmark, the suicide rate is 11.9 per 100,000 people per year according the World Health Organization [1]. Around 8,000 to 10,000 people attempt suicide each year based on information registered in the National Patient Register and the Register of Suicide. Patients who attempted suicide have an increased risk of recurrent suicide attempts and suicidal death [2]. Borderline Personality Disorder (BPD), according to the DSM-IV [3], affects approximately $1 \%$ to $2 \%$ of the general population, and this patient group represents up to $10 \%$ of all psychiatric outpatients and $20 \%$ of all inpatients [2,4]. A total of $60 \%$ to $70 \%$ of the patients with borderline personality disorder will attempt suicide, and the lifetime risk of death by suicide is as much as $10 \%$ [5], a risk 50 times higher than in the general population. Approximately $70 \%$ to $75 \%$ of individuals with BPD, a condition which is associated with challenges in regulating emotions and difficulties in tolerating emotional distress [6], exhibit nonsuicidal self-harm [6].

Dialectical behavior therapy is a manual-based treatment, which was originally developed by the American psychologist Marsha Linehan [6] for the treatment of the core problems of patients with borderline personality disorder, especially concerning emotional regulation, which makes patients particularly prone to self-harming acts. The treatment has shown positive effect in several randomized trials to reduce the high risk of self-injury and suicide attempt in patient with BPD compared with treatment as usual [7-12]. A recent meta-analysis has shown DBT to be the most evidence-based psychotherapeutic treatment for borderline personality disorder and can reduce numbers of self-harming acts and related secondary markers compared with other psychotherapeutic treatments, for example, cognitive behavioral therapy and interpersonal psychotherapy [13]. DBT has also shown effect in treating other dysfunctions such as drug dependence [14] and eating disturbances [15]. A recent systematic review [16] describes seven different studies where the DBT treatment duration varies from 3 to 12 months, and indicates that the most treatment gain is made during the first 4 months of treatment.

The collaborative assessment and management of suicidality (CAMS)-informed supportive psychotherapy was developed by David Jobes and has been somewhat modified in conjunction with Danish colleagues for this study. CAMS is considered both a philosophy of care and a clinical therapeutical framework [17-19]. As a flexible therapeutical framework, CAMS is trans-theoretical and can thereby by used across theoretical orientations, clinical techniques, and professional disciplines. CAMS is designed to specifically target suicidal ideation and behavior as the central clinical focus, independent of diagnosis. Through collaborative assessment and deconstruction of the patient's suicidality, key problems and goals naturally emerge. Collaborative treatment planning that follows thus creates a problem-focused approach that is designed to reconstruct more viable ways of coping and living. Early studies of CAMS have shown positive effects in reducing suicide ideation in smaller studies with suicidal patients on several parameters [19-23], but there is a need for further research in well-powered clinical trials [24].

The two interventions - DBT versus CAMS - have been chosen for the DiaS trial. They have not previously been compared head to head. This trial will target patients with borderline personality traits and recent suicide attempts.

\section{Methods/Design}

Our plan is to compare 16 weeks treatment of DBT versus CAMS. The primary outcome will be the ratio of participants with suicide attempts or non-suicidal deliberate selfharming acts (self-reported and registered in medical records) during the treatment period in the treatment period and at follow-up until week 28 after randomization.

This trial is designed as a single-center, two-armed, parallel-group, observer-blinded randomized clinical superiority trial (Figure 1).

\section{Recruitment and criteria for inclusion and exclusion}

The patients are recruited through the Centre of Excellence for Suicide Prevention, Capital Region of Denmark. This is a highly specialized outpatient care center treating 450 patients annually and offering short-term supportive psychotherapy and social counseling. The patients are referred from general practitioners and from somatic and psychiatric wards after suicide attempts; patients can also self-refer to the Centre. The patients will be referred to the trial and screened by an assessor. If the patients fulfill the inclusion criteria and none of the exclusion criteria (see list of inclusion and exclusion criteria below), they be will included in the trial and randomized by the first contact therapist in the Centre of Excellence for Suicide Prevention. In the screening interview patients will be subjected to a Mini International Neuropsychiatric Interview (MINI) [25] and a Structured Clinical Interview for DSM-IV Axis II Personality Disorders [26]. Deliberate Self-Harm (DSH) and recent suicide attempts will be measured by using Lifetime Suicide and Self-Injury (L-SASI) [27] and Suicide Attempt and Self-injury Interview (SASII) [28]. The therapist will contact the patients and inform them of which group of the treatment they are to be allocated. The adherence to the treatments in both groups will be measured continuously. Follow-up interviews are performed in week 17, 28, and 52 after inclusion

\section{Inclusion and exclusion criteria in the DiaS trial Inclusion criteria}

1) 18 to 65 years of age. 


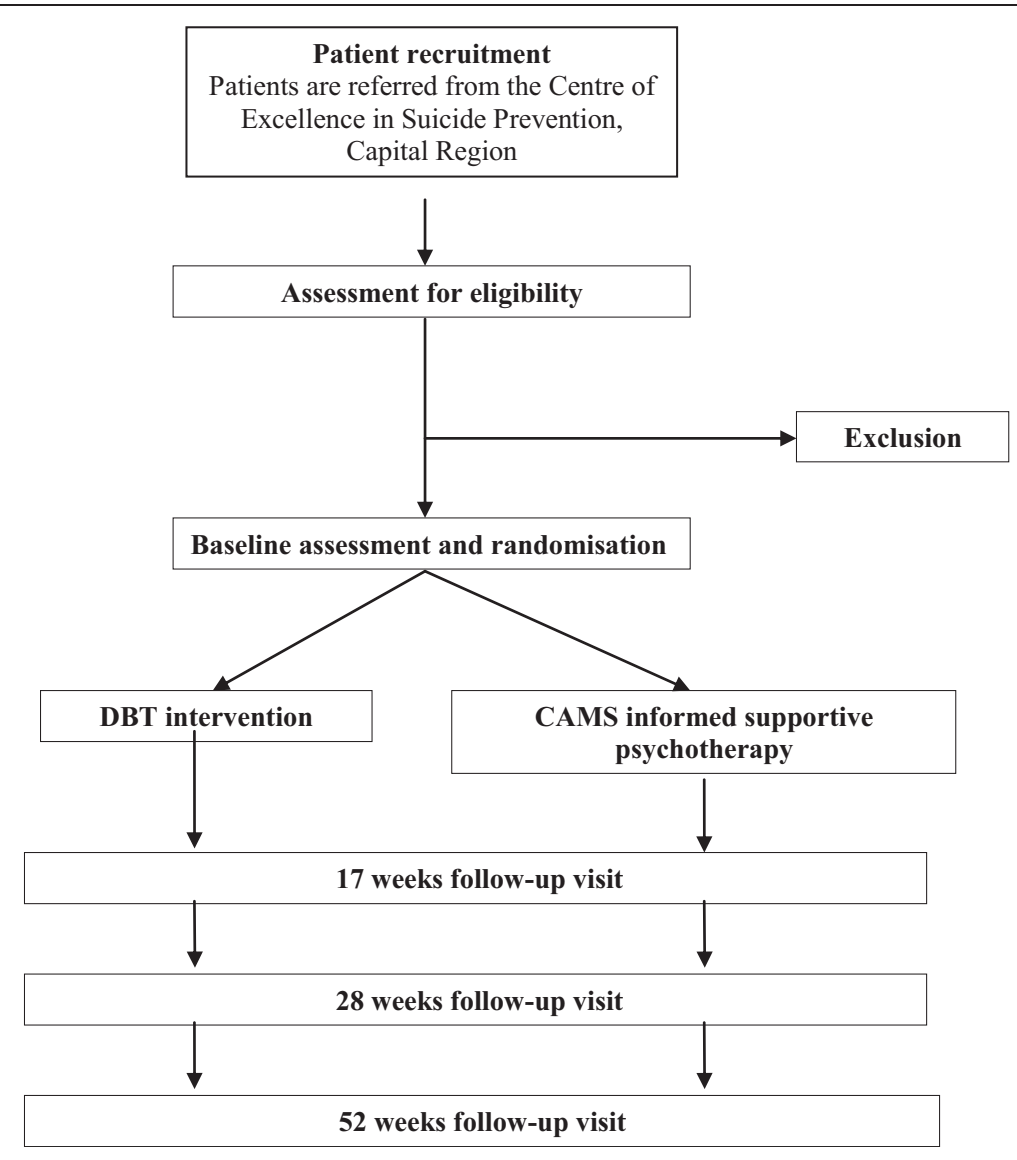

Figure 1 The flowchart of the recruiting and randomization in the DiaS trial.

2) Recent suicide attempt within one month from the inclusion date.

3) At least two of the following criteria for borderline personality disorder (DSM-IV) ${ }^{\text {a }}$ :

a) Desperate efforts to avoid being betrayed or abandoned in reality or in imagination.

b) A pattern of unstable and intense interpersonal relationships characterized by a swinging between extremes of idealization and devaluation.

c) Identity disturbance: markedly and persistently unstable self-image or self-feeling.

d) Impulsivity in at least 2 areas that are potentially self-harming (for example, sexual misconduct, abuse, consumption, and overeating).

e) Repeated instances of suicidal behavior, gestures or threats or self-harm.

f) Affective instability.

g) Chronic feelings of emptiness.

h) Inappropriate intense anger or difficulty controlling anger.

i) Transient, stress related paranoid ideation, delusions or severe dissociative symptoms.

4) Informed consent.

\section{Exclusion criteria}

- Severe depression. ${ }^{\mathrm{b}}$

- Bipolar disorder.

- Psychosis in schizophrenia spectrum. ${ }^{\text {b }}$

- Anorexia nervosa. ${ }^{\mathrm{b}}$

- Alcohol or drug addiction. ${ }^{\mathrm{b}}$

- Mental retardation.

- Dementia.

- Insufficient ability in speaking and understanding Danish

- Lack of informed consent.

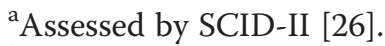

${ }^{\mathrm{b}}$ Assessed by Mini International Neuropsychiatric Interview (MINI) [25].

All psychiatric departments in the Capital region of Denmark have been informed of the DiaS trial through meetings and leaflets (see inclusion criteria above). Patients with at least two traits from the BPD diagnosis (DSM-IV) [3] and a recent suicide attempt are referred through the Centre of Excellence in Suicide Prevention. The suicide attempt has to be within a month from the 
first contact with the Centre. The date has been selected to ensure the accuracy of reporting the intent and other important circumstances (feelings, thoughts and behavior prior to, at the time of, and after the suicide attempt). The patients must speak and understand Danish sufficiently to participate in the therapy sessions and the assessment of the trial. The diagnostic criteria for exclusion have been chosen to eliminate potential confounders in the trial and ensure a homogenous group of patients.

\section{Randomization and blinding}

Patients will be randomized into one of two treatment arms. The randomization is stratified by sex and previous number of self-harm acts (one versus multiple). This is done to avoid the risk of patients with multiple selfharm acts being overrepresented in one of the treatment groups. The Copenhagen Trial Unit (CTU) will generate a computer-generated random sequence randomization, using alternating block sizes unknown to the investigators. The trial staff will contact the CTU for information about allocation. The above-mentioned procedure ensures adequate allocation concealment. The patients and therapists will not be blinded. We will use blinded outcome assessors (see outcomes below).

\section{Dialectical behavior therapy}

Dialectical behavior therapy (DBT) consists of four components: 1) individual therapy, 2) skills training in groups, 3) access to telephone contact with therapists, and 4) supervision and consultations for the therapist to prevent burn-out. The first aim of the therapy is organized around a target hierarchy, which consists of 1) eliminating lifethreatening behavior including suicide attempts and deliberate self-harm, 2) eliminating treatment interfering behavior such as non-attendance and not doing homework, and 3) ameliorating behaviors leading to a decreased quality of life (axis I disorders, social aspects, and drug dependence). The second aim of the therapy is skill training in groups. Patients are taught four modules of primary skills: 1) mindfulness, 2) emotional regulation, 3) distress tolerance, and 4) interpersonal effectiveness. The individual therapy will focus on the taught skills in the groups. The DBT treatment manual is based on DBT-A by Rathus and Miller [29]. The DBT intervention will consist of 16 weeks treatment defined as 2 hours weekly skill training in groups, 1 hour weekly individual psychotherapy, opportunity of telephone coaching with the therapist, and approximately 1 hour weekly team consultation and supervision.

\section{Collaborative assessment and management of suicidality-informed supportive psychotherapy} The second intervention consists of supportive psychotherapeutic treatment within the framework of CAMS.
CAMS-informed supportive psychotherapy is a psychotherapeutic approach based on principles from the 'Collaborative assessment and management of suicidality' [19]. The method typically employs a 45- to 60-minute session once a week in the office of the therapist (in crisis situations the length, frequency, and location of care may vary). CAMS sessions have a specific structure starting in the first 10 to 20 minutes with a collaborative working through of the 'core assessment' of the 'Suicide Status Form' (SSF), rating on a scale of 1 to 5 the following six suicide-related markers: psychological pain, stress, agitation, hopelessness, self-hate, and overall risk of suicide. In the collaborative exploration of these and additional SSF constructs, the patient and the therapist sit side-by-side. Following the initial assessment, various 'drivers' of suicidality are identified and further investigated. Direct drivers are thoughts, feelings, behaviors and interpersonal themes that lead to suicidal thoughts and acts. Indirect drivers are other factors that contribute but do not directly lead to suicidal ideation or feelings, such as: unemployment, depression, substance abuse, post-traumatic stress disorder. A treatment plan is collaboratively formulated that emphasizes a 'crisis response plan' to establish outpatient stability. In addition, the CAMS treatment plan further identifies, targets, and treats suicidal drivers with problem-focused interventions. On-going CAMS care involves the development of coping skills for emergencies (for example, the elaboration and use of a crisis response plan) as well as the on-going linking of drivers and suicidality and problemfocused interventions that treat those issues that makes the patient suicidal. This philosophical and clinical approach to treatment is used in subsequent CAMS sessions as the problem-focused treatment to ultimately eliminate suicidal coping as much as possible.

The duration of the CAMS treatment differs depending on the patient's suicidality. The therapist concludes CAMS treatment after three successive contracts where the patient is assessed to be non-suicidal according to the SSF criteria [17]. In this study, the therapists are supervised one and a half hours every second week.

\section{Program fidelity}

The trial staff consists of four clinical psychologists, two nurses, a social worker, and an occupational therapist. All have had courses and are trained in DBT and CAMSinformed supportive psychotherapy. The training courses offered the therapists were conducted by Alan Fruzzetti, Professor at University of Reno, who trained the staff for 10 days in an intensive DBT training course that consisted of four modules spread over a time period of 8 months and by David Jobes, who held a 2-day course twice on CAMS-informed supportive psychotherapy. The individual sessions with the patients will be videotaped and used 
to rate the adherences in both interventions. In the DBT group the rating scale to be used will be the DBT-global rating scale by Marsha Linehan (unpublished work). The therapist adherence to CAMS-informed supportive psychotherapy will be assessed with the CAMS rating scale (DA Jobes, unpublished work).

The CAMS intervention will be carried out at the established departments of the Centre of Excellence for Suicide Prevention, Capital Region of Denmark, Mental Health Services, Psychiatric Centre of Copenhagen and Psychiatric Centre of Amager. The DBT intervention will be carried out at the Centre of Excellence for Suicide Prevention, Psychiatric Centre of Copenhagen.

\section{Assessments}

The patients will be subjected to four almost identical assessments. The first baseline assessment will be performed in continuity with the assessment and validation of the inclusion and exclusion criteria. Also the strata used for the randomization will be obtained in the first interview with the patient. The second assessment will occur after treatment has ended in week 17 after inclusion in the trial. The third and fourth assessment will occur at a follow-up interview after week 28 and week 52 after inclusion.

\section{Outcomes}

\section{Primary outcome}

The primary outcome will be measured as the ratio of deliberate self-harm before and after treatment (week 0 compared with week 28). The Suicide attempt and SelfInjury Interview (SASII) [28] will be used to collect these data and direct questions in the follow-up interview regarding self-harming behavior after inclusion in the trial. We will also obtain information concerning numbers of deliberate self-harm and suicide attempts through medical records.

\section{Explorative outcomes}

In our trial we have chosen a number of exploratory outcomes, which are not based on power calculations. These include the severity of BPD symptoms, suicide intention and ideation, depression, hopelessness, self-esteem, impulsivity, and anger. We will also examine between-group differences in 'dosage' and durations of the respective treatments.

\section{Borderline personality disorder symptoms}

The severity of features regarding borderline personality symptoms is measured by the Zanarinis Rating Scale for BPD. It is a nine-item, five-point scale ( 0 to $4,0=$ no symptoms and $4=$ severe symptoms) validated, clinicianbased diagnostic interview (DSM-IV) [30]. The categories of symptoms are affective, cognitive, impulsivity, and interpersonal symptoms.

\section{Suicide intention and ideation}

Suicide intention is measured by Beck's Suicide Intent Scale (SIS) [31,32]. It consists of 20 items, where the different circumstances and practical preparations are investigated.

The Beck Suicide Ideation Scale (BSS) is a 19-item self-report questionnaire measuring suicidal thinking [32]. Items are scored on a Likert scale ranging from 0 to 2 , with a higher score indicating more severe suicidal ideation.

\section{Depression}

Depressive symptoms will be assessed by Hamilton Depression Rating Scale (HDRS) 17-items [33]. The patients are also subjects to Beck's Depression Inventory (BDI) $[34,35]$, a 21 -item self-report inventory of the depressive symptomatology measuring depression. The BDI is the most widely used research instruments and the items are rated on a Likert scale from 0 to 3, where higher scores indicate a more severe level of depressive symptoms.

\section{Hopelessness, self-esteem, impulsivity, anger}

The Becks Hopelessness Scale (BHS) consists of 20 truefalse items pertaining to future outlook [36]. Rosenberg's Self- Esteem scale (RSE) is used to assess the self-esteem. It consists of 10 items and is a Likert scale with answers using a four-point scale -from strongly agree to strongly disagree [37].

Barratts Impulsivity Scale 11 (BIS-11) is a 30-item questionnaire measuring impulsive personality traits [38]. It yields a total score, three second-order factors, and six first-order factors [39]. To measure anger, we will use State Trait Anger Scale (STAS), developed by Charles Spielberger [40]. It consists of 20 items in a selfreport questionnaire and is rated on a Likert scale with answers using a four-point scale -from never to always.

\section{Sample size and statistical analyses}

Based on previous estimations, we expect the risk of renewed self-harming acts within their first year to be 50 percent in the CAMS treatment group [6]. We expect the DBT to be able to reduce this risk to 25 percent, a relative risk reduction of $50 \%$. With a power of $90 \%$ and a type one error probability associated with this test of 0.05 , we plan to randomize 154 patients, with about 77 patients in each intervention group. We will handle missing data using logistic regression with multiple imputations as described below.

The two group baseline characteristics will be compared using Student's $t$-test for independent samples or a chi-square test for binary variables.

The primary analysis will be based on the intentionto-treat principle; that is, data from all patients will be included in the treatment group to which they were allocated regardless of degree of compliance. 
Binary outcomes will be analyzed using logistic regression and continuous outcomes will be analyzed using linear regression with baseline variables as sex and previous deliberate self-harm as a covariates.

If more than $5 \%$ of the primary outcome is missing at follow-up, we will use multiple imputations (SPSS version 19.0). If this is the case, the multiple imputations will be considered the primary result. For multiple imputations, we will use a linear regression model with 100 imputations and 20 iterations. The pooled estimates from the imputations will subsequently be used for our analysis.

We will use two-tailed tests for statistical significance with alpha set at $P<0.05$.

\section{Ethical considerations}

The trial protocol is approved by the regional ethics committee in the Capital Region of Denmark under file number H-1-2011-042. The Danish Data Protection Agency has approved the management of data in the trial under the file number 2007-58-0015. Finally, the trial is registered under ClinicalTrial.gov as NCT01512602. In accordance with the CONSORT guidelines [41], we will publish negative, neutral and positive findings in the trial.

We have decided not to have a control group without any intervention, because of the ethical aspects when treating suicidal and vulnerable patients.

The patients will be informed about the trial with both verbally and with written information before signing the written consent. It will be stressed that participation in the trial is voluntary. Participation and the written consent can be withdrawn at any time in the trial, and this can be done with no consequence for future treatment possibilities.

\section{Discussion}

The DiaS trial is designed as a pragmatic trial, with the intention of intervening in the exiting clinical outpatient setting, so one could argue the patient population is selected beforehand. The Excellence Centre for Suicide Prevention has their exclusion criteria before offering treatment as mentioned before and can exclude patients for whom treatment can appear too demanding. The chosen target group includes patients with emotional dysregulation leading to suicide attempts. This patient group is over-represented in the Centre of Excellence in Suicide Prevention. The chosen experimental DBT treatment is based on a modified DBT manual but where all the skills taught are maintained. The DBT treatment period of 16 weeks is shorter than in the majority of other randomized clinical trials performed [16]. The considerations of these modulations are the excepted occurrence of the lesser chronicity and severity in BPD symptoms in the target group of patients. The patients only have to fulfill two out of nine BPD criteria according to the DSM-IV. Regarding the CAMS-informed supportive psychotherapy given to the control group, this treatment has been an obvious choice as the majority of therapist in the Excellence Centre for Suicide Prevention use this treatment. This treatment group can be considered as an optimized 'treatment-as-usual' control group, where the therapists are trained and supervised, and treatment adherence is rated.

The two different intervention groups will receive different amounts of time of individual psychotherapy, and the CAMS group is not offered skill training in groups. We consider the different amounts of time in the two groups as inevitable because of the different basic construction of the two psychotherapeutic treatments. In the CAMS- intervention group the therapist has to finish the treatment when achieving three consecutive nonsuicidal scores on the SSF. A strength of this trial is the centralized randomization [42], which reduces the risk of selection bias. We will perform blinded outcome assessment to reduce bias [43]. We will use intentionto-treat analysis and multiple imputations to reduce the bias introduced by missing data in our analysis [43].

A related post-hoc consideration is the potential 'costeffectiveness' of the respective treatments in relation to frequencies of hospitalizations, emergency department visits, and primary care visits. The cost-effectiveness of clinical care for high-risk patients was previously examined in a retrospective study of CAMS versus treatmentas-usual with suicidal outpatients [44] and is a topic of increasing importance within contemporary mental health care around the world.

The DiaS trial is a single center trial and can therefore be considered as a pilot trial for multicenter trials in the future. In order to obtain external validity, we need multicenter trials. The DiaS trial has a 52-week follow-up period to investigate deliberate self-harm and suicide attempts after treatment. This knowledge can be useful in the design of trials, in regard to treatment duration or to booster treatment sessions. Moreover we need trials showing comparable effects both during the intervention period and the post-intervention period.

\section{Trial status}

The status of the trial is recruitment of patients, which began in January 2012 and is expected to finish in January 2014. We must recruit 154 participants, and we find this goal realistic because it is estimated that approximately $30 \%$ of the patients in the target group at the Excellence Centre for Suicidal Prevention will fulfill the inclusion criteria for the DiaS trial.

\section{Abbreviations}

BDI: Beck's depression inventory; BPD: borderline personality disorder; BHS: Beck's hopelessness scale; BIS-11: Barratt's impulsivity scale 11; BSS: Beck's suicide ideation scale; CAMS: collaborative assessment and 
management of suicidality; CTU: Copenhagen trial unit; DBT: dialectical behavior therapy; DBT-A: dialectical behavior therapy-adolescent; DSH: self liberate self-harm; DSM-IV: Diagnostic and Statistical Manual of Mental Disorders-4th edition; L-SASI: lifetime suicide and self-injury interview; HDRS-17: Hamilton depression rating scale-17; RSE: Rosenberg's self-esteem scale; SASIl: suicide and self-injury interview; SIS: Beck's suicide intent scale; SSF: suicide status form; SPSS: Statistical Package for the Social Sciences.

\section{Competing interests}

The authors declare that they have no competing interests.

\section{Authors' contributions}

KTA was responsible for the study conception and the design of the trial protocol, and writing the manuscript. MN was responsible for study conception and the design of the trial protocol, writing the manuscript and critical revision of the work. JK participated in the design of the trial, writing the manuscript, and critical revision of the work. BR participated in writing the manuscript and critical revision of the work. CG participated in the design of the trial, writing the manuscript and critical revision of the work. DJ participated in writing the manuscript and critical revision of the work. All authors read and approved the final manuscript.

\section{Acknowledgements}

The trial has received funding from the Lundbeck Foundation and The Capital Region of Denmark's strategic research foundation. The Faculty of Health, University of Copenhagen and The Capital Region of Copenhagen have given KTA a Ph.D. scholarship. Neither current nor future sponsors of the trial will have any role in the trial design, data collection, data analysis, data interpretation, or publication of data from the trial.

\section{Author details}

${ }^{1}$ Mental Health Centre Copenhagen, Faculty of Health Science, University of Copenhagen, Copenhagen, Denmark. ${ }^{2}$ Mental Health Services, Copenhagen and Faculty of Health Sciences, University of Copenhagen, Copenhagen, Denmark. ${ }^{3}$ Copenhagen Trial Unit, Centre for Clinical Intervention Research, Rigshopitalet, Copenhagen University Hospital, DK-2100 Copenhagen, Denmark. ${ }^{4}$ Department of Psychology, The Catholic University of America, Washington, DC, USA. ${ }^{5}$ Research Unit, Mental Health Centre, Bispebjerg bakke 23, Building 14, DK-2400 Copenhagen, NV, Denmark.

Received: 6 September 2013 Accepted: 16 May 2014

Published: 29 May 2014

\section{References}

1. Suicide rates (per 100.000), by gender Denmark, 1950-2006- World Health Organization. In [http://www.who.int/mental_health/media/denm.pdf]

2. Owens D, Horrocks J, House A: Fatal and non-fatal repetition of self-harm. Systematic review. Br J Psychiatry 2002, 181:193-199.

3. American Psychiatric Association: Diagnostic and Statistical Manual of Mental Health Disorders. 4th edition. Washington DC: APA; 1994.

4. Lieb K, Zanarini MC, Schmahl C, Linehan MM, Bohus M: Borderline personality disorder. Lancet 2004, 364:453-461.

5. Gunderson JG: Borderline Personality Disorder: A Clinical Guide. Washington DC: American Psychiatric Press; 2001

6. Linehan MM: Cognitive Behavioral Treatment of Borderline Personality Disorder. New York: Guilford Press; 1993.

7. Linehan MM, Armstrong HE, Suarez A, Allmon D, Heard HL: Cognitive-behavioral treatment of chronically parasuicidal borderline patients. Arch Gen Psychiatry 1991, 48:1060-1064

8. Linehan MM: Dialectical behavior therapy for treatment of borderline personality disorder: implication for the treatment of substance abuse. NIDA Res Monogr 1993, 137:201-216.

9. Linehan MM, Comtois KA, Murray AM, Brown MZ, Gallop RJ, Heard HL, Korslund KE, Tutek DA, Reynolds SK, Lindenboim N: Two-year randomized controlled trial and follow-up of dialectical behavior therapy vs therapy by experts for suicidal behaviors and borderline personality disorder. Arch Gen Psychiatry 2006, 63:757-766.
10. Van den Bosch LM, Verheul R, Schippers GM, Van den Brink W: Dialectical behavior therapy of borderline patients with and without substance use problems. Implementation and long-term effects. Addict Behav 2002, 27:911-923.

11. Van den Bosch LM, Koeter MW, Stijnen T, Verheul R, Van den Brink W: Sustained efficacy of dialectical behaviour therapy for borderline personality disorder. Behav Res Ther 2005, 43:1231-1241.

12. Verheul $R$, Van Den Bosch LM, Koeter MW, De Ridder MA, Stijnen T, Van Den Brink W: Dialectical behaviour therapy for women with borderline personality disorder: 12-month, randomised clinical trial in The Netherlands. Br J Psychiatry 2003, 182:135-140.

13. Stoffers JM, Vollm BA, Rucker G, Timmer A, Huband N, Lieb K: Psychological therapies for people with borderline personality disorder. Cochrane Database Syst Rev 2012, 8:CD005652.

14. Linehan MM, Schmidt H 3rd, Dimeff LA, Craft JC, Kanter J, Comtois KA Dialectical behavior therapy for patients with borderline personality disorder and drug-dependence. Am J Addict 1999, 8:279-292.

15. Chen EY, Matthews L, Allen C, Kuo JR, Linehan MM: Dialectical behavior therapy for clients with binge-eating disorder or bulimia nervosa and borderline personality disorder. Int J Eat Disord 2008, 41:505-512.

16. Rizvi SL, Linehan MM: Dialectical behavior therapy for personality disorders. Curr Psychiatry Rep 2001, 3:64-69.

17. Jobes DA, Drozd JF: The CAMS approach to working with suicidal patients. J Contemp Psychother 2004, 34:73-85.

18. Jobes DA: Collaborating to prevent suicide: a clinical-research perspective. Suicide Life Threat Behav 2000, 30:8-17.

19. Jobes DA: Managing Suicidal Risk; A Collaborative Approach. New York: Guilford Press; 2006

20. Ellis TE, Green KL, Allen JG, Jobes DA, Nadorff MR: Collaborative assessment and management of suicidality in an inpatient setting: results of a pilot study. Psychotherapy (Chic) 2012, 49:72-80.

21. Comtois KA, Jobes DA, O'Connor SS, Atkins DC, Janis K, Chessen CE, Landes SJ, Holen A, Youdelis Flores C: Collaborative assessment and management of suicidality (CAMS): feasibility trial for next-day appointment services. Depress Anxiety 2011, 28:963-972.

22. Arkov K, Rosenbaum B, Christiansen L, Jonsson H, Munchow M: Treatment of suicidal patients: the collaborative assessment and management of suicidality. Ugeskr Laeger 2008, 170:149-153.

23. Nielsen AC, Alberdi F, Rosenbaum B: Collaborative assessment and management of suicidality method shows effect. Dan Med Bull 2011, 58:A4300.

24. Jobes DA: The collaborative assessment and management of suicidality (CAMS): an evolving evidence-based clinical approach to suicidal risk. Suicide Life Threat Behav 2012, 42:640-653.

25. Sheehan DV, Lecrubier $Y$, Sheehan KH, Amorim P, Janavs J, Weiller E, Hergueta T, Baker R, Dunbar GC: The mini-international neuropsychiatric interview (M.I.N.I.): the development and validation of a structured diagnostic psychiatric interview for DSM-IV and ICD-10. J Clin Psychiatry 1998, 59(Suppl 20):22.

26. First MB, Gibbon M, Spitzer RL, Wiliams JBW, Benjamin LS: Structured Clinical Interview for DSM-IV Axis II Personality Disorders (SCID-II). Washington DC: American Psychiatric Press, Inc.; 1997.

27. Linehan MM, Comtois K: Lifetime Parasuicide History. ; 1996.

28. Linehan MM, Comtois KA, Brown MZ, Heard HL, Wagner A: Suicide attempt self-injury interview (SASII): development, reliability, and validity of a scale to assess suicide attempts and intentional self-injury. Psychol Assess 2006, 18:303-312

29. Rathus JH, Miller AL: Dialectical behavior therapy adapted for suicidal adolescents. Suicide Life Threat Behav 2002, 32:146-157.

30. Zanarini MC, Vujanovic AA, Parachini EA, Boulanger JL, Frankenburg FR, Hennen J: Zanarini rating scale for borderline personality disorder (ZAN-BPD): a continuous measure of DSM-IV borderline psychopathology. J Pers Disord 2003, 17:233-242.

31. Beck AT, Schyler D, Herman L: Development of suicidal intent scales. In The Prediction of Suicide. Edited by Beck AT, Resnik HLP, Lettieri D. Bowie, Maryland: Charles Press; 1974:45-46.

32. Beck AT, Kovacs M, Weissman A: Assessment of suicidal intention: the scale for suicide ideation. J Consult Clin Psychol 1979, 47:343-352.

33. Hamilton M: A rating scale for depression. J Neurol Neurosurg Psychiatry 1960, 23:56-62.

34. Beck AT, Ward CH, Mendelson M, Mock J, Erbaugh J: An inventory for measuring depression. Arch Gen Psychiatry 1961, 4:561-571. 
35. Beck AT, Rush AJ, Shaw BF: Cognitive Therapy of Depression. New York: Guilford Press; 1979

36. Beck AT, Steer RA: Manual for Becks Hopelessness Scale. Tex, Psychological Corp.: San Antonio; 1988

37. Rosenberg M: Society and the Adolescent Self-image. Princeton, NJ: Princeton University Press; 1965.

38. Barratt ES: Factor analysis of some psychometric measures of impulsiveness and anxiety. Psychol Rep 1965, 16:547-554.

39. Patton JH, Stanford MS, Barratt ES: Factor structure of the Barratt impulsiveness scale. J Clin Psychol 1995, 51:768-774.

40. Spielberg CD, Jacobs G, Russell S, Crane R: Assessment of anger: The State-Trait Anger Scale. In Advances in Personality Assessment (vol 2). Edited by Butcher JN. Spielberg CD. Hillsdale, NJ: LEA; 1983.

41. Consort. In [www.consort-statement.org]

42. Wood L, Egger M, Gluud LL, Schulz KF, Jüni P, Altman DG, Gluud C, Martin RM, Wood AJG, Sterne JAC: Empirical evidence of bias in treatment effect estimates in controlled trials with different interventions and outcomes: meta-epidemiological study. BMJ 2008, 336:601-605.

43. Little RJ, D'Agostino R, Cohen ML, Dickersin K, Emerson SS, Farrar JT, Frangakis C, Hogan JW, Molenberghs G, Murphy SA, Neatin JD, Rotnitzky A, Scharfstein D, Shih WJ, Siegel JP, Stern H: The prevention and treatment of missing data in clinical trials. N Engl J Med 2012, 367:1355-1360.

44. Jobes DA, Wong SA, Conrad AK, Drozd JF, Neal-Walden T: The collaborative assessment and management of suicidality versus treatment as usual: a retrospective study with suicidal outpatients. Suicide Life Threat Behav 2005, 35:483-497.

doi:10.1186/1745-6215-15-194

Cite this article as: Andreasson et al:: The DiaS trial: dialectical behavior therapy versus collaborative assessment and management of suicidality on self-harm in patients with a recent suicide attempt and borderline personality disorder traits - study protocol for a randomized controlled trial. Trials 2014 15:194.

\section{Submit your next manuscript to BioMed Central and take full advantage of:}

- Convenient online submission

- Thorough peer review

- No space constraints or color figure charges

- Immediate publication on acceptance

- Inclusion in PubMed, CAS, Scopus and Google Scholar

- Research which is freely available for redistribution 